\title{
Novel biodegradable magnesium alloy clips compared with titanium clips for hepatectomy in a rat model
}

\author{
Takeshi Urade ${ }^{1 *}$ (D), Toshihiko Yoshida ${ }^{1}$, Naoko Ikeo², Kosuke Naka², Masahiro Kido', Hirochika Toyama', \\ Kimihiko Ueno ${ }^{1}$, Motofumi Tanaka', Toshiji Mukai ${ }^{2}$ and Takumi Fukumoto ${ }^{1}$
}

\begin{abstract}
Background: The use of surgical metal clips is crucial for ligating vessels in various operations. The currently available metal clips have several drawbacks; they are permanent and interfere with imaging techniques such as computed tomography (CT) or magnetic resonance (MR) imaging and carry the potential risk of endo-clip migration. We recently developed a novel magnesium (Mg) alloy for biodegradable clips that reduces artifacts on $\mathrm{CT}$ imaging. This study aimed to examine the tolerance, biodegradability, and biocompatibility of the Mg alloy clips compared with those of standard titanium (Ti) clips in hepatectomy.
\end{abstract}

Methods: Thirty Wistar rats were divided into two groups based on the clip used (groups A and B). The vascular pedicle, including hepatic artery, portal vein, bile duct, and hepatic vein of the left lateral lobe, was ligated with the Ti clip in group A or the Mg alloy clip in group B, and then the left lateral lobe was removed. The rats were sacrificed at 1 , $4,12,24$, and 36 weeks after surgery. Clinical and histological evaluations were performed. Absorption rate was calculated by measuring the clip volume.

Results: Although the Mg alloy clips showed biodegradability over time, there were no significant differences in the serum concentration of $\mathrm{Mg}$ between the two groups. The remaining volume ratio of $\mathrm{Mg}$ alloy clips was 95.5, 94.3, 80.0, 36.2 , and $16.7 \%$ at $1,4,12,24$, and 36 weeks, respectively. No side effects occurred. Most of the microscopic changes were similar in both groups.

Conclusions: The new biodegradable Mg alloy clips are safe and feasible in vessel ligation for hepatectomy in a rat model and reduce artifacts in CT imaging compared with the standard Ti clips.

Keywords: Biodegradable clip, Absorbable clip, Magnesium clip, Metal clip, Hepatectomy, Rat

\section{Background}

The use of surgical metal clips is crucial for ligating vessels in various operations. The currently available metal clips are made of pure titanium (Ti) and $\mathrm{Ti}$ alloys, which are strong and ductile enough to occlude vessels in soft tissues using forceps. However, Ti clips have several drawbacks; they form metallic artifacts, which hamper the image quality in computed tomography $(\mathrm{CT})$ evaluations, and they permanently remain in the human body,

\footnotetext{
* Correspondence: uradet1125@gmail.com

${ }^{1}$ Department of Surgery, Division of Hepato-Biliary-Pancreatic Surgery, Kobe University Graduate School of Medicine, 7-5-2 Kusunoki-cho, Chuo-ku, Kobe 650-0017, Japan

Full list of author information is available at the end of the article
}

sometimes resulting in adhesion or endo-clip penetration and migration. [1-3] Some patients develop allergic reactions to $\mathrm{Ti}$ ions. [4] Absorbable polymer clips were developed in the early 1990s, [5] but the use of polymer clips is limited due to their thickness and weakness of clipping force.

Recently, magnesium $(\mathrm{Mg})$ alloys have attracted considerable attention because of their excellent biocompatibility and biodegradability. Biodegradable instruments made of Mg alloys are used in bone screws, orthopedic implants, and dental implants used for oral maxillofacial surgery. [6-10] However, the physical properties of the Mg alloys necessary for use as operative clips differ from those of other instruments. Besides suitable biodegradability, it

(c) The Author(s). 2019 Open Access This article is distributed under the terms of the Creative Commons Attribution 4.0 International License (http://creativecommons.org/licenses/by/4.0/), which permits unrestricted use, distribution, and reproduction in any medium, provided you give appropriate credit to the original author(s) and the source, provide a link to the Creative Commons license, and indicate if changes were made. The Creative Commons Public Domain Dedication waiver (http://creativecommons.org/publicdomain/zero/1.0/) applies to the data made available in this article, unless otherwise stated. 
requires sufficient ductility and mechanical strength for enabling tissue ligation. Considering this, we developed a novel metal clip made of $\mathrm{Mg}$ alloys, which has enough strength for ligation, biodegradability, and reduces artifacts on CT imaging. [11, 12]

In liver surgery, hepatic transection is a key determinant of intraoperative blood loss and postoperative complications. During hepatic transection, surgical metal clips are frequently used to ligate the biliary and vascular structures to reduce surgery time. [13] In a previous study, we evaluated the feasibility of our $\mathrm{Mg}$ alloy clip in a canine cholecystectomy model. [12] However, we have not yet evaluated the influence of the $\mathrm{Mg}$ alloy clip on the vascular structure. The aims of this study were to confirm the ability of the Mg alloy clip for vessel sealing, to evaluate the serum concentration of $\mathrm{Mg}$, and to check the degradation behavior when we used the Mg alloy clips for vessel ligation in hepatectomy. A rat model was used in the present study because numerous previous studies have already established the hepatectomy method and it is easy to perform CT scan repeatedly to evaluate the degradation of the clips.

\section{Methods}

This study was conducted in accordance with the Kobe University Animal Experimentation Regulation and was approved by the Institutional Animal Care and Use Committee (permission number: P141203).

\section{Materials}

The manufacturing method of the new Mg alloy clip has been described in our previous study. [11] This $\mathrm{Mg}$ alloy consists of $99.8 \% \mathrm{Mg}, 0.2 \%$ zinc $(\mathrm{Zn})$, and $0.1 \%$ calcium (Ca); it has sufficient mechanical strength and ductility for enabling tissue ligation by controlling its microstructure. A clip with a cross-section of $0.7 \times 0.7 \mathrm{~mm}^{2}$ and a side $6 \mathrm{~mm}$ long was fabricated using hot extrusion. The clip was machined from the bar followed by an acid wash. The acid used in this study was $5 \%$ Nital solution ( $5 \%$ nitric acid $+95 \%$ ethanol). The weight after the acid wash of the clip was unified to be approximately $11 \mathrm{mg}$. A Ti clip (LIGACLIP Extra Ligating Clip, Medium, Ethicon Endo-Surgery, LLC, Cincinnati, OH) was used as a control. The clip applier (Multi-Patient Single-Clip Applier, Ethicon Endo-Surgery, LLC) was used to apply the $\mathrm{Ti}$ and $\mathrm{Mg}$ alloy clips (Fig. 1a and b).

\section{Animal model and surgical procedure}

Thirty male healthy Wistar rats (8 weeks old; weight, $310-380 \mathrm{~g}$ ) were divided into two groups: group A was treated with $\mathrm{Mg}$ alloy clips $(n=15)$, whereas group B was treated with Ti clips $(n=15)$. The rats were supplied by Charles River Laboratories Japan, Inc. (Yokohama, Japan). Two rats were placed inside each cage in a temperature-controlled room, with food and water provided ad libitum under a 12-h light/dark diurnal cycle. Operations were conducted under general anesthesia with isoflurane. The animals were placed in a supine position, and laparotomy was performed with a midline abdominal incision. After the median lobe was retracted to the cranial side and the caudate lobe to the caudal side, the vascular pedicle, including hepatic artery, portal vein, bile duct, and hepatic vein of the left lateral lobe, was encircled. Lastly, the pedicle was ligated using the $\mathrm{Ti}$ or Mg alloy clip, and the left lateral lobe was removed (Fig. 2). After complete hemostasis was confirmed, the abdominal wall was closed. Three rats in each group were humanely euthanized with exsanguination under deep anesthesia in the laboratory, followed by extraction of the clips at $1,4,12,24$, or 36 weeks after surgery.

\section{Blood chemistry}

Blood biochemical testing was conducted to evaluate concentrations of aspartate aminotransferase (AST), alanine aminotransferase (ALT), creatinine (Cre), calcium (Ca), and Mg. Blood samples were taken from the caudal vein preoperatively and at $1,4,12$, and 24 weeks postoperatively in the 24-week observation group rats (group A: $n=3$, group B: $n=3$ ) and were measured by ORIENTAL YEAST Co., Ltd. (Tokyo, Japan).
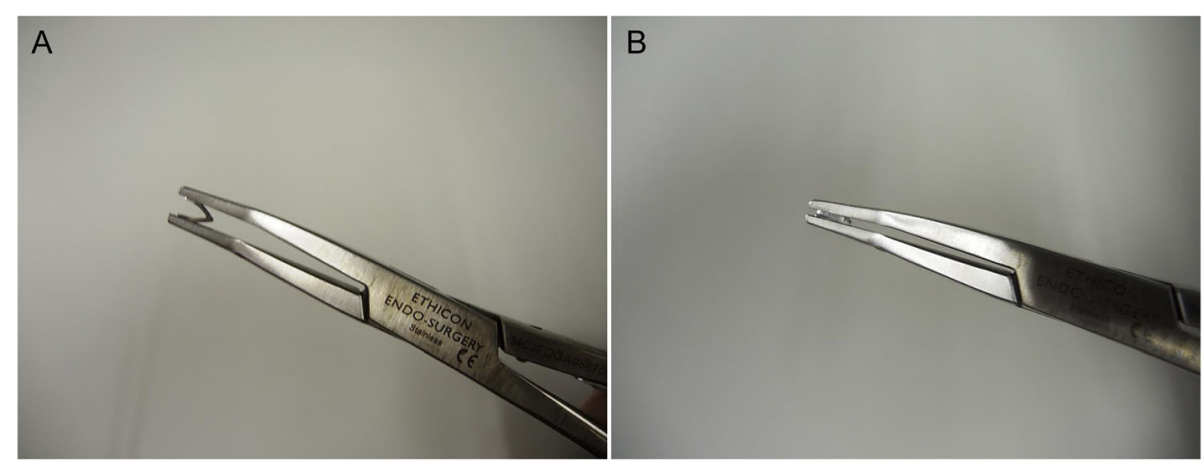

Fig. 1 Photograph of a magnesium alloy clip. a A magnesium alloy clip mounted on the clip applier. b A closed magnesium alloy clip 


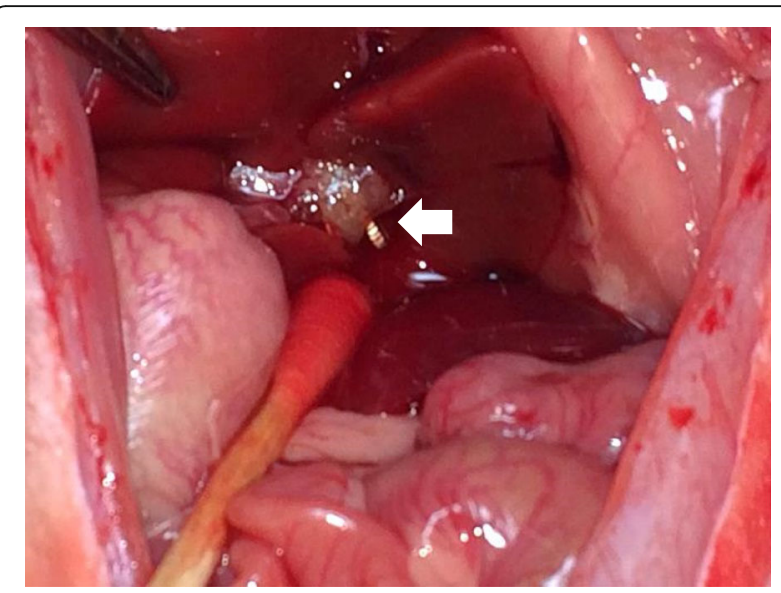

Fig. 2 Photograph of the operative field after hepatectomy. The left lateral lobe was removed using the Mg alloy clip (white arrow)

\section{Micro-CT imaging}

Micro-CT imaging using an in vivo micro-CT scanning machine (R_mCT2, Rigaku, Japan) was also performed under general anesthesia with isoflurane. The reaction of the intra-abdominal implanted clips was evaluated just after surgery and at 1, 4, 12, 24, and 36 weeks postoperatively in the 36 -week observation group rats (group A: $n=3$, group B: $n=3$ ).

\section{Estimation of metallic artifact}

Both metallic clips formed metallic artifacts that interfered with image quality in CT. The artifacts' appearances were evaluated on $\mathrm{CT}$ images.

\section{Estimation of degradation volume}

The clips were extracted at the end of the observation period at 1, 4, 12, 24, and 36 weeks after surgery in group A $(n=15)$. To estimate the degradation rate, the remaining ratio, which was the volume after extraction divided by the volume before implantation, was calculated. The volume before implantation was estimated from the division of the weight by the alloy density. The volume after extraction was evaluated from the three-dimensional CT data obtained by micro-focus X-ray computerized tomography ( $\mu \mathrm{CT}$; ScanX-mate-E90, Comscantecno Co., Ltd., Yokohama, Japan).

\section{Histologic analysis}

The tissue, including hepatocytes around clips, was extracted and fixed in 10\% formalin, embedded in paraffin, and cut into 3-mm thick slices. Hematoxylin and eosin staining was performed to evaluate the degree of inflammatory reaction.

\section{Statistical analysis}

Blood chemistry data were compared between the two groups using $t$-test. A $p$ value $<0.05$ was considered statistically significant. All calculations were performed with the help of JMP software version 10 (SAS Institute, Cary, NC, USA).

\section{Results}

\section{Surgical outcomes}

All rats survived surgery and no significant complications occurred throughout the observation period. No intra-abdominal hemorrhage was observed, and all clips were found in the implanted place and the vascular pedicle was closed on micro-CT images.

\section{Investigation of blood parameters}

The blood test data of the 24-week observation group rats (group A: $n=3$, group B: $\mathrm{n}=3$ ) are shown in Fig. 3 . The serum AST and ALT levels gradually increased after surgery, but the AST and ALT levels were comparable between the two groups (Fig. 3a and b). In addition, there were no significant differences in the serum concentrations of $\mathrm{Mg}$ and $\mathrm{Ca}$, which are components of the $\mathrm{Mg}$ alloy clips in both groups (Fig. $3 \mathrm{~d}$ and e). There was a significant difference in the Cre level at 1 week after implantation (Fig. 3c).

\section{Micro-CT imaging data analysis}

Figure 4 shows CT images of the rats in both groups just after surgery. Strong artifacts were formed radially from the Ti clips, as shown in Fig. 4a. Conversely, few artifacts were formed from the $\mathrm{Mg}$ alloy clips throughout the observation period, as shown in Fig. 4b.

\section{Ability for closure of the vascular pedicle}

Figure 5 shows the chronological change in the Mg alloy clips from just after surgery to 36 weeks in the 36-week observation group rats (group A: $n=3$, group B: $n=3$ ). The form of the $\mathrm{Mg}$ alloy clip was maintained within 4 weeks (Fig. $5 \mathrm{a}-\mathrm{C}$ ). Afterward, the $\mathrm{Mg}$ alloy clip became completely thin at 12 weeks (Fig. $5 \mathrm{~d}$ ) and split into two at 24 weeks (Fig. 5e). Finally, it was dismantled at 36 weeks (Fig. 5f). According to the CT images, the ability for closure of the vascular pedicle was confirmed until 12 weeks at least.

\section{Change in the volume of $\mathrm{mg}$ alloy clips}

The Mg alloy clips in 15 rats (group A: $n=15$ ) were extracted and observed using micro-CT scanning to determine their degradation behavior. The 3D images reconstructed from the CT images are shown in Fig. 6a-e and correspond to $1,4,12,24$, and 36 weeks after surgery, respectively. The longer the clips were implanted, the thinner and smaller they became. Figure $6 \mathrm{f}$ shows that the 


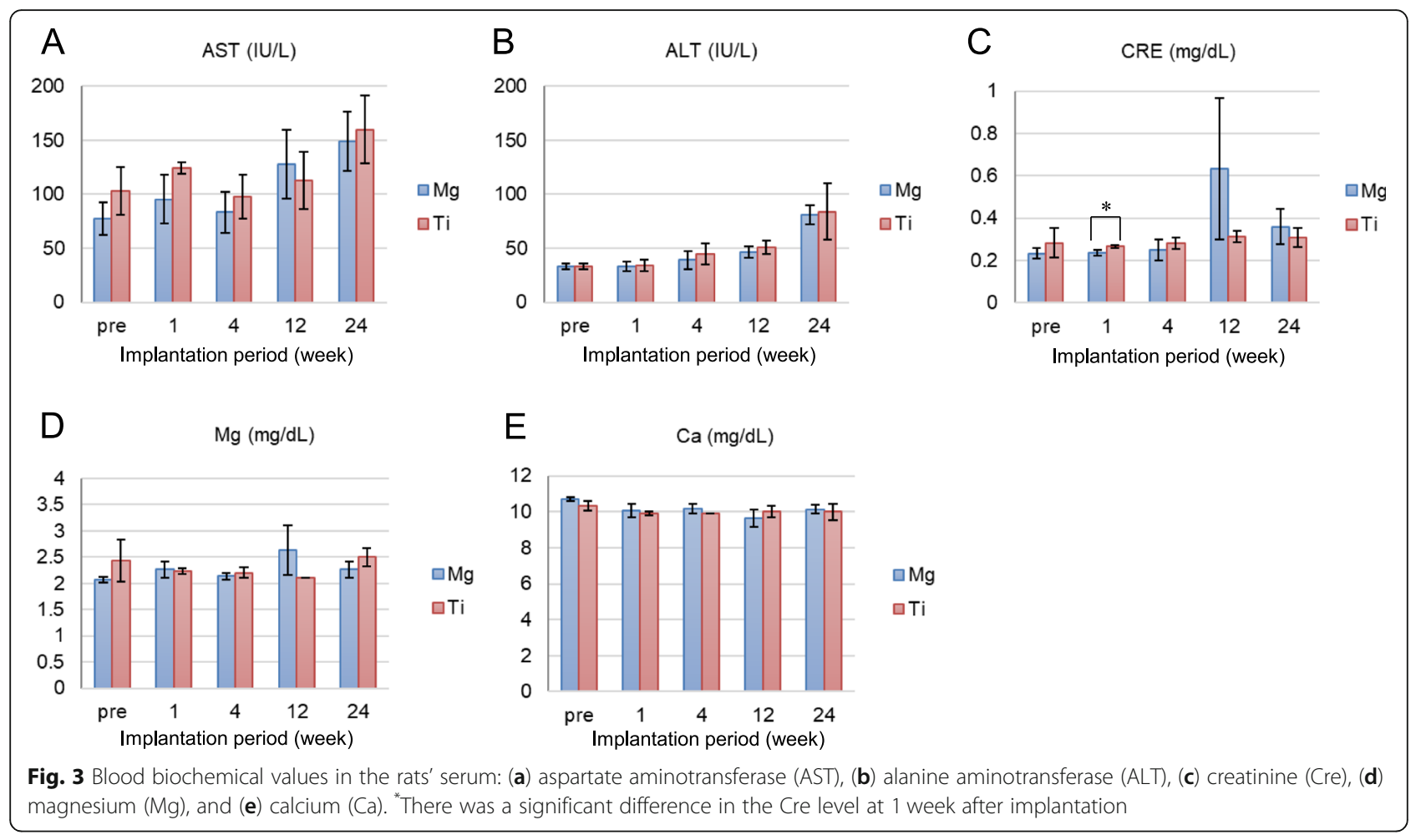

calculated remaining volume ratio of the Mg alloy clip decreased gradually during the observation period. The remaining volume ratio of $\mathrm{Mg}$ alloy clips was 95.5, 94.3, 80.0, 36.2, and $16.7 \%$ at $1,4,12,24$, and 36 weeks, respectively.

\section{Histologic findings}

Hematoxylin and eosin staining of the tissue, including hepatocytes, around the clips at 12 weeks is shown in Fig. 7. Mild inflammation and fibrosis could be seen in the tissue around the clips in both groups (Fig. 7a and b). No significant difference was observed between both groups.

\section{Discussion}

This study showed that our Mg alloy clips are feasible and sufficiently tolerable biodegradable surgical clips for vessel sealing during hepatectomy. In addition, there was no evidence that the serum concentration of $\mathrm{Mg}$ elevated throughout the observation period.

Our $\mathrm{Mg}$ alloy clips possessed sufficient properties for the 10-min occlusion of a renal vein in a rat model, and for the 24-week occlusion of a cystic duct in a canine model. [11, 12] However, the hemostatic performance and the degradation behavior of our $\mathrm{Mg}$ alloy clips after vessel sealing for a long observation period has not been evaluated yet. When we apply the Mg alloy clip for vessel sealing, we should consider the effects of the blood

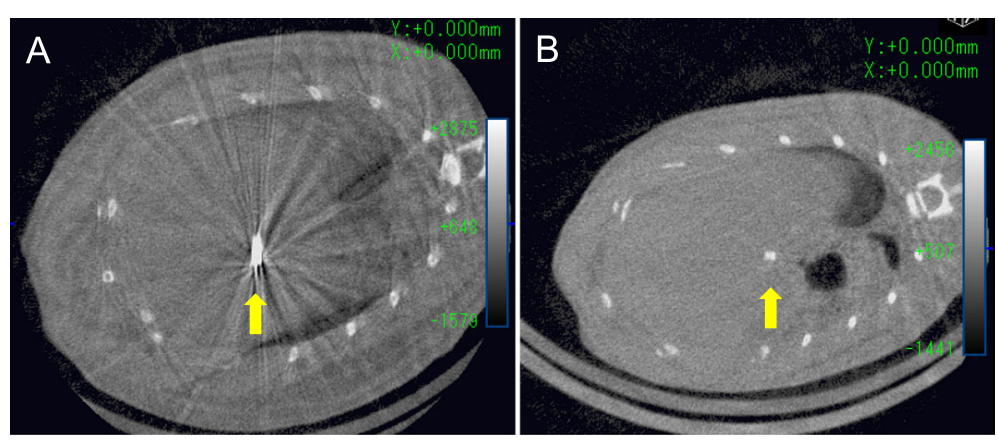

Fig. 4 Computed tomography images just after hepatectomy. a Strong artifacts were formed radially from the titanium clip (yellow arrow). b Few artifacts were formed from the magnesium alloy clip (yellow arrow) 

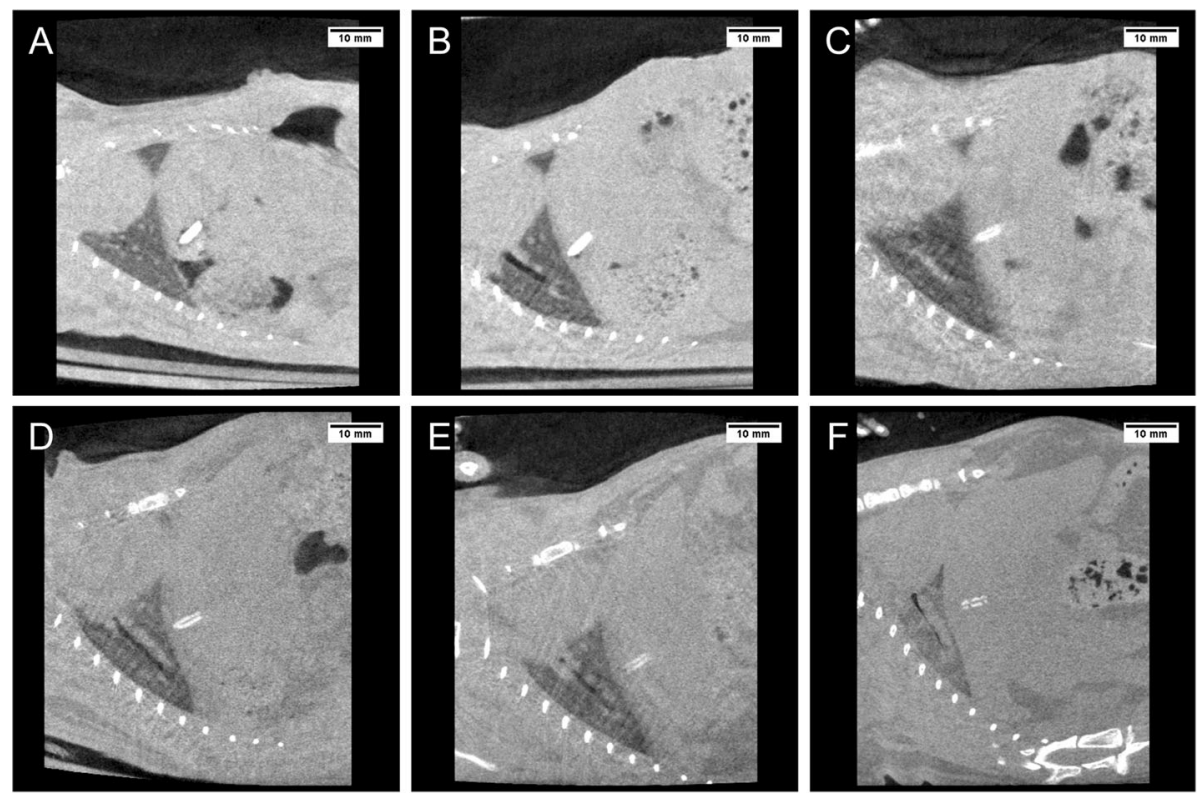

Fig. 5 Computed tomography images of the same rat after hepatectomy using the magnesium alloy clip: (a) just after hepatectomy, (b) after 1 week, (c) after 4 weeks, (d) after 12 weeks, (e) after 24 weeks, and (f) after 36 weeks

flow on the clip. After tightening the clip, it might cause absorption from the blood vessel wall or come in direct contact with the blood flow of capillary vessels. These conditions may induce rapid degradation of the clip, considerable hydrogen gas production, and elevation of serum $\mathrm{Mg}$ levels resulting in failure of vessel sealing. In this study, we first applied our Mg alloy clip for vessel sealing in rat hepatectomy and demonstrated that our $\mathrm{Mg}$ alloy clips possess sufficient characteristics for vessel sealing during hepatectomy.
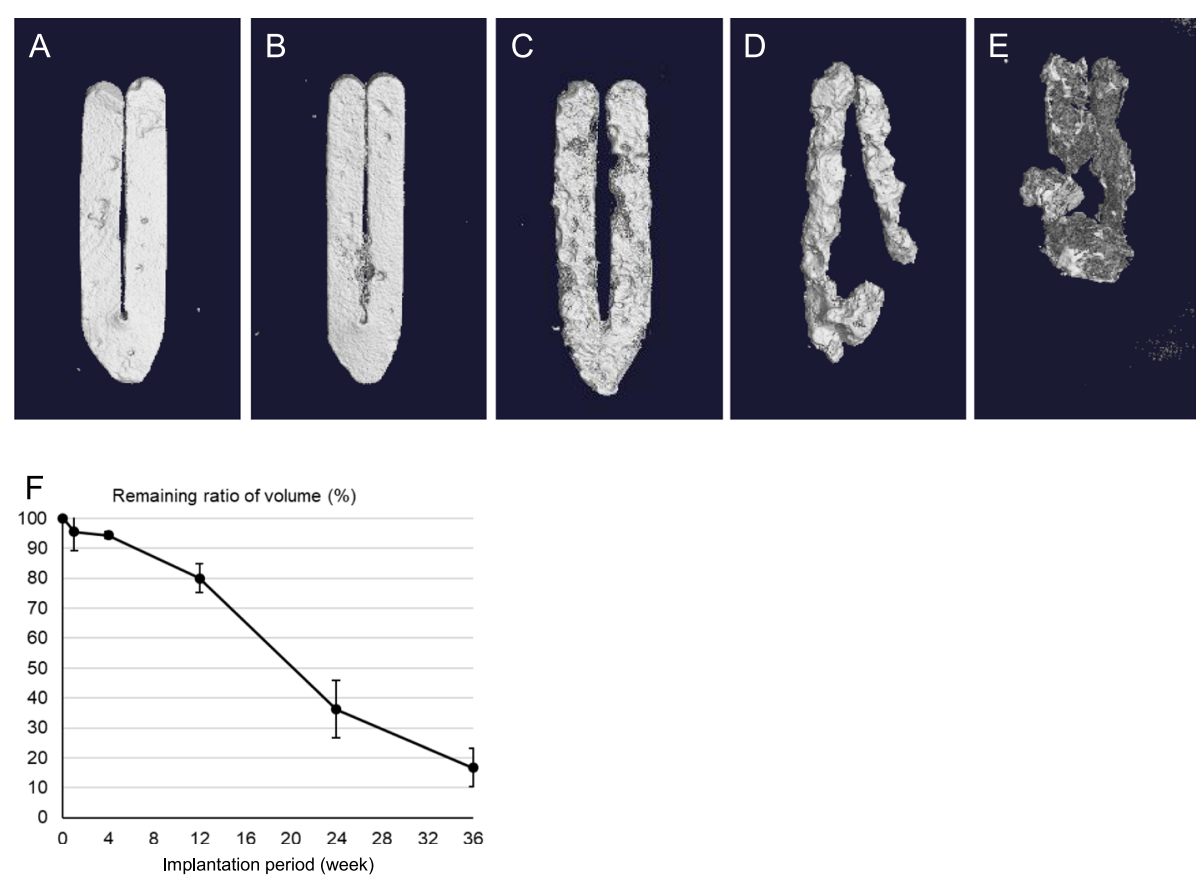

Fig. 6 Three-dimensional computed tomography images of the magnesium alloy clip in the same rat after hepatectomy: (a) after 1 week, (b) after 4 weeks, (c) after 12 weeks, (d) after 24 weeks, and (e) after 36 weeks. The relationship between the remaining volume ratio and the implantation period is shown in (f) 

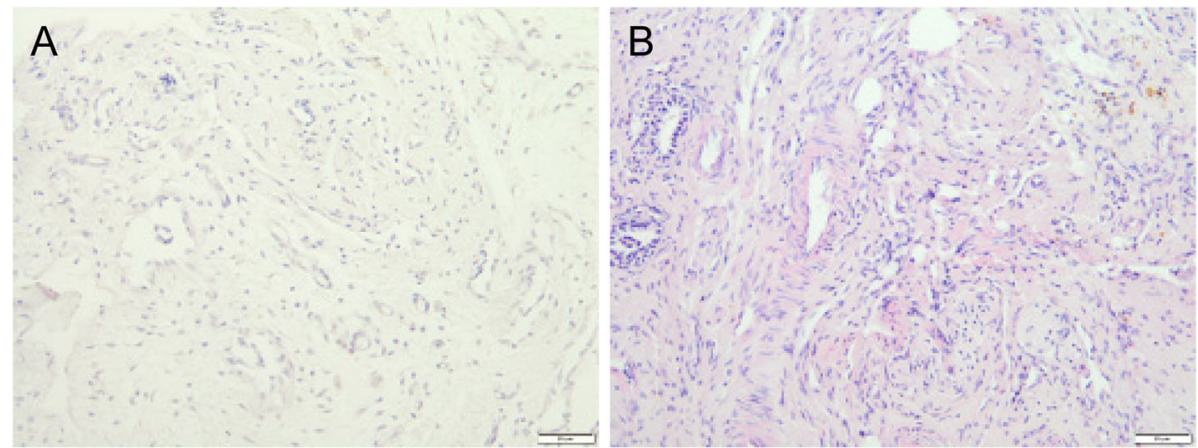

Fig. 7 Hematoxylin and eosin staining of the tissue, including hepatocytes, around the clips at 12 weeks. a Titanium clip and (b) magnesium alloy clip. Scale bar: $20 \mu \mathrm{m}$

Pure Mg has excellent biodegradability in a physiological environment. However, in turn, this high degradation rate of pure $\mathrm{Mg}$ and the resulting evolution of hydrogen gas are the main obstacles to clinical application as a surgical clip. To regulate the degradation velocity, several previous studies devised methods of coating using other materials such as gold, silicon, and polymer; these coating methods resulted in slow degradation. [14-18] However, the surface coating is in danger of peeling off through mechanical irritation during surgery or postoperatively. Moreover, it may trigger localized degradation through crevice corrosion. Therefore, we need to develop new ways to overcome this in vivo instability. Our Mg alloy clips showed continuous biodegradability in the abdominal cavity and approximately $64 \%$ of them were absorbed at 24 weeks (Fig. 6). This is slower than that seen in the canine cholecystectomy model; approximately $87 \%$ of the clips were absorbed at 24 weeks. This difference in biodegradability may be affected by the differences among animal species, targets of $\mathrm{Mg}$ alloy clips and circumstances of their placement. Altogether, we believe that this degradation speed is sufficient for vessel sealing because the clot formation in the vessel occurred early after tightening the clip.

Metallic artifacts that interfere with CT and magnetic resonance imaging are problematic. Modern CT units can reduce metallic artifacts induced by $\mathrm{Ti}$ or stainless steel by using advanced technologies. However, they result in reduced image quality [19] or high peak voltage of $x$-ray radiation. [20] Previous studies reported that replacing metal clips with polymeric absorbable clips reduce the artifacts. [21] However, currently available polymer clips have several disadvantages compared with metal clips. Since they are thicker and larger than metal clips, surgeons feel that it is difficult to close vessels without the sufficient margin of clipping. In addition, it is difficult to remove them because they have a rigid outer polyglycolic body or locking system. In contrast, our previous studies demonstrated that $\mathrm{Mg}$ alloy clips form fewer metallic artifacts than that with Ti clips. [12] Moreover, this study demonstrated that an $\mathrm{Mg}$ alloy clip maintains its shape for 12 weeks, the volume of the clip gradually decreases because of its biodegradability, and that metallic artifacts eventually completely disappear. Thus, visualization of ligation after using clips with minimal artifacts provides a sense of security when postoperative CT is performed to determine the cause of complications, at least in the perioperative period. Conversely, disappearance of the clips facilitates accurate diagnosis of recurrence of cancers without artifacts in the late postoperative phase.

In liver surgery, many vascular structures need to be ligated and divided during parenchymal transection. Over the last three decades, open and laparoscopic liver resection has been increasingly performed for the treatment of liver diseases, but the type of instruments by energy sources and technique used for hepatic transection vary among institutions. [22, 23] Although various vessel-sealing devices and ultrasonic scalpels have been developed and widely used, whether vascular systems, including bile ducts and thick vessels, are completely occluded with these devices has not been proven. Conversely, clip ligation of biliary and vascular structures is a safe and time-saving technique compared to ligation with thread. [13] Thus, we believe that Mg alloy clips are useful for ligation of biliary and vascular structures during hepatectomy. Additionally, surgeons who prefer metal clips to polymeric clips would believe that our $\mathrm{Mg}$ alloy clips close thin vessels relatively easily and remove themselves in mis-ligation cases similar to Ti clips.

The price of $\mathrm{Mg}$ alloys is an important issue. The market prices of raw $\mathrm{Mg}$ is approximately less than one third of that of $\mathrm{Ti}$, and the melting temperatures of $\mathrm{Ti}$ and $\mathrm{Mg}$ are $1941 \mathrm{~K}$ and $923 \mathrm{~K}$, respectively. These facts indicate that the fabrication cost of the present $\mathrm{Mg}-\mathrm{Zn}-\mathrm{Ca}$ alloy is considerably lower than that of conventional $\mathrm{Ti}$ alloy for medical application (e.g., Ti-6Al-4 V alloy). Therefore, we expect that the production cost of our $\mathrm{Mg}$ 
alloy clips would be lower than that of currently available Ti clips. Furthermore, the deformation behavior of our $\mathrm{Mg}$ alloy is relatively similar to that of $\mathrm{Ti}$, compared with a polymer material, due to the work-hardening property and/or plastic deformation capability. This suggests that the $\mathrm{Mg}$ alloy clips can be used with conventional clip dispensers. We believe that this ability also contributes to the total production cost.

The main limitation of this study is that we only used a minimum number of healthy small animals for hepatectomy. Hepatectomy for patients with liver tumors requires much more ligation of vessels and leads to more intraoperative bleeding and postoperative ascites that might influence the degradation behavior of the $\mathrm{Mg}$ alloy clips. Thus, further examinations, including hepatectomy, in large animals are required to apply the $\mathrm{Mg}$ alloy clips in clinical situations.

In this study, we revealed that $\mathrm{Mg}$ alloy clips enabled closure of vessels with good hemostasis. In addition, there was no evidence that the serum Mg concentration elevated, and the degradation behavior was not problematic when we applied the biodegradable $\mathrm{Mg}$ alloy clips for vessel ligation in rat hepatectomy.

\section{Conclusions}

This study aimed to examine the tolerability and safety of the novel biodegradable $\mathrm{Mg}$ alloy clips compared with those of standard $\mathrm{Ti}$ clips for hepatectomy in a rat model. These Mg alloy clips have the potential to replace standard metal clips in the near future. However, further studies are needed to investigate the use of biodegradable Mg alloy clips for the same indication in large animals as well as in clinical human models.

\section{Abbreviations}

ALT: Alanine aminotransferase; AST: Aspartate aminotransferase; Ca: calcium Cre: creatinine; CT: Computed tomography; Mg: magnesium; MR: Magnetic resonance; Ti: titanium; $\mathrm{Zn}$ : zinc

\section{Acknowledgements}

We thank Dr. Tomoo Ito and Dr. Masayuki Akita for their great efforts in evaluating the histologic findings. We would like to thank Editage (www.editage.jp) for English language editing.

This study was presented at the 54th Congress of the European Society for Surgical Research, Geneva, Switzerland, March 2019. [24]

\section{Authors' contributions}

TU and TF contributed to the study conception and design. TU, TY, NI, and KN acquired the data. TU, TY, NI, KN, MK, HT, KU, and MT analyzed and interpreted the data. TU, TM and TF drafted the manuscript. TU, NI, TM, and TF critically revised the manuscript. All authors approved the final version of the manuscript.

\section{Funding}

This study was supported by Hyogo Center of Excellence Program Promotion Project in the design of the study; The Japan Society for the Promotion of Science (JSPS KAKENHI grant numbers JP16K10426 and JP17H01327) in data collection, analysis and interpretation.

\section{Availability of data and materials}

The datasets generated and/or analyzed during the current study are available from the corresponding author on reasonable request.

\section{Ethics approval and consent to participate}

This study was conducted in accordance with the Kobe University Animal Experimentation Regulation and was approved by the Institutional Animal Care and Use Committee (permission number: P141203).

\section{Consent for publication}

Not applicable.

\section{Competing interests}

The authors declare that they have no competing interests.

\section{Author details}

${ }^{1}$ Department of Surgery, Division of Hepato-Biliary-Pancreatic Surgery, Kobe University Graduate School of Medicine, 7-5-2 Kusunoki-cho, Chuo-ku, Kobe 650-0017, Japan. ²Department of Mechanical Engineering, Kobe University, Kobe, Japan.

Received: 5 January 2019 Accepted: 2 September 2019

Published online: 09 September 2019

\section{References}

1. Matsuura T, Kanisawa Y, Sato T, Saito T, Hirata K. Migration of "endo-clips" into common bile-duct after laparoscopic cholecystectomy. Lancet. 1992; 340:306.

2. Sato T, Denno R, Yuyama Y, Matsuura T, Kanisawa Y, Hirata K. Unusual complications caused by endo-clip migration following a laparoscopic cholecystectomy: report of a case. Surg Today. 1994;24:360-2.

3. Matsumoto H, Ikeda E, Mitsunaga S, Naitoh M, Furutani S, Nawa S. Choledochal stenosis and lithiasis caused by penetration and migration of surgical metal clips. J Hepato-Biliary-Pancreat Surg. 2000;7:603-5.

4. Müller K, Valentine-Thon E. Hypersensitivity to titanium: clinical and laboratory evidence. Neuro Endocrinol Lett. 2006;27(Suppl 1):31-5.

5. Klein RD, Jessup G, Ahari F, Connolly RJ, Schwaitzberg SD. Comparison of titanium and absorbable polymeric surgical clips for use in laparoscopic cholecystectomy. Surg Endosc. 1994:8:753-8.

6. Akmaz I, Kiral A, Pehlivan O, Mahirogullari M, Solakoglu C, Rodop O. Biodegradable implants in the treatment of scaphoid nonunions. Int Orthop. 2004;28:261-6.

7. Stener S, Ejerhed L, Sernert N, Laxdal G, Rostgård-Christensen L, Kartus J. A long-term, prospective, randomized study comparing biodegradable and metal interference screws in anterior cruciate ligament reconstruction surgery: radiographic results and clinical outcome. Am J Sports Med. 2010; 38:1598-605.

8. Bashti K, Tahmasebi MN, Kaseb H, Farahmand F, Akbar M, Mobini A Biomechanical comparison between Bashti bone plug technique and biodegradable screw for fixation of grafts in ligament surgery. Arch Bone Jt Surg. 2015;3:29-34.

9. Abdal-Hay A, Hasan A, Kim YK, Lee MH, Hamdy AS, Khalil KA Biocorrosion behavior of biodegradable nanocomposite fibers coated layer-by-layer on AM50 magnesium implant. Mater Sci Eng C Mater Biol Appl. 2016:58:1232-41.

10. Brunner S, Vavken P, Kilger R, Vavken J, Rutz E, Brunner R, et al. Absorbable and non-absorbable suture fixation results in similar outcomes for tibial eminence fractures in children and adolescents. Knee Surg Sports Traumatol Arthrosc. 2016:24:723-9.

11. Ikeo N, Nakamura R, Naka K, Hashimoto T, Yoshida T, Urade T, et al. Fabrication of a magnesium alloy with excellent ductility for biodegradable clips. Acta Biomater. 2016;29:468-76.

12. Yoshida T, Fukumoto T, Urade T, Kido M, Toyama H, Asari S, et al. Development of a new biodegradable operative clip made of a magnesium alloy: evaluation of its safety and tolerability for canine cholecystectomy. Surgery. 2017;161:1553-60.

13. Haberal M, Emiroğlu R, Karakayali H, Arslan G, Turan C, Bilgin N. Silver clip ligation technique for liver resection. Transplant Proc. 2001:33:2030-1.

14. Xin Y, Jiang J, Huo K, Tang G, Tian X, Chu PK. Corrosion resistance and cytocompatibility of biodegradable surgical magnesium alloy coated with hydrogenated amorphous silicon. J Biomed Mater Res A. 2009;89:717-26. 
15. Wang J, He Y, Maitz MF, Collins B, Xiong K, Guo L, et al. A surface-eroding poly (1,3-trimethylene carbonate) coating for fully biodegradable magnesium-based stent applications: toward better biofunction, biodegradation and biocompatibility. Acta Biomater. 2013:9:8678-89.

16. Zomorodian A, Garcia MP, Moura e Silva T, Fernandes JC, Fernandes MH, Montemor MF. Corrosion resistance of a composite polymeric coating applied on biodegradable AZ31 magnesium alloy. Acta Biomater. 2013;9: 8660-70.

17. Ostrowski N, Lee B, Enick N, Carlson B, Kunjukunju S, Roy A, et al. Corrosion protection and improved cytocompatibility of biodegradable polymeric layer-by-layer coatings on AZ31 magnesium alloys. Acta Biomater. 2013;9: 8704-13.

18. Chng CB, Lau DP, Choo JQ, Chui CK. A bioabsorbable microclip for laryngeal microsurgery: design and evaluation. Acta Biomater. 2012;8:2835-44.

19. Liu PT, Pavlicek WP, Peter MB, Spangehl MJ, Roberts CC, Paden RG. Metal artifact reduction image reconstruction algorithm for $\mathrm{CT}$ of implanted metal orthopedic devices: a work in progress. Skelet Radiol. 2009;38:797-802.

20. Lee MJ, Kim S, Lee SA, Song HT, Huh YM, Kim DH, et al. Overcoming artifacts from metallic orthopedic implants at high-field-strength MR imaging and multi-detector CT. Radiographics. 2007;27:791-803.

21. Westwood TD, Capozzi P, Martin DF, Sukumar SA. Absorbable laparoscopic ligating clips: impact on postoperative CT imaging. Clin Radiol. 2014:69:783-5.

22. Lesurtel M, Selzner M, Petrowsky H, McCormack L, Clavien PA. How should transection of the liver be performed?: a prospective randomized study in 100 consecutive patients: comparing four different transection strategies. Ann Surg. 2005;242:814-22, discussion 22-3.

23. Otsuka Y, Kaneko H, Cleary SP, Buell JF, Cai X, Wakabayashi G. What is the best technique in parenchymal transection in laparoscopic liver resection? Comprehensive review for the clinical question on the 2 nd international consensus conference on laparoscopic liver resection. J Hepatobiliary Pancreat Sci. 2015;22:363-70.

24. European Society for Surgical Research, 54th Congress, Geneva, March 2019: Abstracts. Eur Surg Res 2019;60(suppl 2):1-104.

\section{Publisher's Note}

Springer Nature remains neutral with regard to jurisdictional claims in published maps and institutional affiliations.

Ready to submit your research? Choose BMC and benefit from:

- fast, convenient online submission

- thorough peer review by experienced researchers in your field

- rapid publication on acceptance

- support for research data, including large and complex data types

- gold Open Access which fosters wider collaboration and increased citations

- maximum visibility for your research: over $100 \mathrm{M}$ website views per year

At $\mathrm{BMC}$, research is always in progress.

Learn more biomedcentral.com/submissions 